\title{
Special Issue: Pathophysiology and Therapeutic Perspectives in DMD: The Well-Defined Role of the Immune System
}

\author{
Andrea Farini (iD \\ Laboratorio di Cellule Staminali, Dipartimento di Fisiopatologia Medico-Chirurgica e dei Trapianti, \\ Università degli Studi di Milano, Fondazione IRCCS Cà Granda Ospedale Maggiore Policlinico, Milano, \\ Centro Dino Ferrari, Via Francesco Sforza 35, 20122 Milan, Italy; farini.andrea@gmail.com
}

Citation: Farini, A. Special Issue: Pathophysiology and Therapeutic Perspectives in DMD: The Well-Defined Role of the Immune System. Biomedicines 2021, 9, 1911. https: / / doi.org/10.3390/ biomedicines 9121911

Received: 24 November 2021 Accepted: 1 December 2021 Published: 14 December 2021

Publisher's Note: MDPI stays neutral with regard to jurisdictional claims in published maps and institutional affiliations.

Copyright: (C) 2021 by the author. Licensee MDPI, Basel, Switzerland. This article is an open access article distributed under the terms and conditions of the Creative Commons Attribution (CC BY) license (https:/ creativecommons.org/licenses/by/ $4.0 /)$.
Duchenne muscular dystrophy (DMD) is the most common, lethal, muscle-wasting disease of childhood. Dystrophin absence alters the contraction machinery and, due to fiber degeneration/regeneration cycles, dystrophic muscles develop fibrosis as a consequence of continuous muscular injury and regenerative potential exhaustion. Together with the loss of sarcolemma stability and the increase in muscular weakness, this condition determines cardiac/respiratory failure and premature death. Although the primary defects rely on skeletal muscle structure, a multitude of secondary defects exist involving metabolic - as well as inflammatory-deregulated pathways. Indeed, immune cell infiltration into the skeletal muscle is a notable feature of the disease pathophysiology and is strongly associated with disease severity.

Immunosuppressive drugs, such as glucocorticoids, are the only effective therapy to delay the onset and control symptoms in a number of patients, but they cause serious side adverse effects, limiting their use in patients. Consistently, a deeper understanding of inflammation-driven mechanisms is fundamental to produce new feasible treatments. In this Special Issue, we reviewed the latest studies on DMD pathogenesis to assess the complex relationship between innate and adaptive immune responses in dystrophic skeletal muscles [1] and open the way for the identification of new therapeutic targets. In particular, Tulangekar et al. described how neutrophils' secretion of myeloperoxidase (MPO) and neutrophil elastase (NE) improved the rise in oxidative stress and consequently the development of inflammation, suggesting their modulation as feasible clinical outcomes [2] Similarly, Herbelet and co-workers investigated the protein kinase A/mitogen-activated protein kinase/nuclear factor of the activated T-cells 5/organic osmolytes (PKA-p38MAPKNFAT5-organic osmolytes) pathway and its role in mediating muscle homeostasis and skeletal muscle regeneration [3].

Regarding the advent of new therapeutic treatments to improve the condition of disabled patients, Testa et al. suggested that the cell-based reconstructive approach could be a feasible approach to circumvent chronic dysfunctions in healing processes that affect DMD muscles [4].

With the same effort to find the optimal muscle progenitor source that show both myogenic potential and high proliferation rates to obtain a sufficient amount of cells, Shen et al. demonstrated that activated mesenchymal stem cells (MSCs) improved the function of distal skeletal muscles following implantation in dystrophic animal models [5]. Similarly, Park et al. demonstrated that the injection of MSCs isolated from Wharton's jelly in mdx mice resulted in the amelioration of a pathological phenotype, as they found the downregulation of $\mathrm{CK}$, apoptosis, and fibrosis, probably through the inhibition of miR-499-5p and its targets, TGF $\beta$ R-1 and -3 [5].

Interestingly, Uchimura and Sakurai obtained differentiated myotubes from DMD patient-derived induced pluripotent stem cells (iPSCs) and showed that the modulation of the activity of calcium channels STIM1 and Orai1 rescued the contractile properties of dystrophic muscles [6]. Dubinin et al. studied the efficacy of alisporivir, a nonimmunosuppressive inhibitor of mitochondrial permeability transition pore (MPTP), to 
modulate the cardiac phenotype of mdx mice, and they found that mitochondrial biogenesis was inhibited, and the amount of mtDNA was markedly reduced [7].

It is well accepted that the most efficacious therapy for DMD should attempt to block the immune responses that are provoked by the inflammatory milieu and preserve the reparative functions in myogenesis that are normally exerted by inflammation. At the same time, other therapeutic interventions are needed to rescue the effects of non-inflammatory dysfunctions, such as those related to mitochondria or calcium influx, that dramatically worsen myofibers' degeneration.

In this regard, in this Special Issue, it was suggested that early and precise combined interventions with regard to inflammatory pathways that affect the skeletal muscle of DMD patients are mandatory both to enhance the feasibility of gene therapy and to allow safer cellular transplantation.

Funding: This study was supported by the Associazione Centro Dino Ferrari, a French Telethon AFM grant (No. 21104) and presents independent research funded by Ricerca Finalizzata 2016 (Linea di ricerca: "Theory-enhancing"). AF was supported by Fondazione IRCCS Cà Granda, Ospedale Maggiore Policlinico $5 \times 1000$ Research Award. YT acknowledges support by Fondo Europeo di Sviluppo Regionale 2014-2020. POR FESR 2014-2020, Ricerca Innovazione and Gruppo familiari beta-sarcoglicanopatie, PR-0394, GFB-ONLUS.

Conflicts of Interest: The authors declare no conflict of interest. The funders had no role in the design of the study; in the collection, analyses, or interpretation of data; in the writing of the manuscript, or in the decision to publish the results.

\section{References}

1. Tripodi, L.; Villa, C.; Molinaro, D.; Torrente, Y.; Farini, A. The Immune System in Duchenne Muscular Dystrophy Pathogenesis. Biomedicines 2021, 9, 1447. [CrossRef] [PubMed]

2. Tulangekar, A.; Sztal, T.E. Inflammation in Duchenne Muscular Dystrophy-Exploring the Role of Neutrophils in Muscle Damage and Regeneration. Biomedicines 2021, 9, 1366. [CrossRef] [PubMed]

3. Herbelet, S.; Merckx, C.; De Paepe, B. The PKA-p38MAPK-NFAT5-Organic Osmolytes Pathway in Duchenne Muscular Dystrophy: From Essential Player in Osmotic Homeostasis, Inflammation and Skeletal Muscle Regeneration to Therapeutic Target. Biomedicines 2021, 9, 350. [CrossRef] [PubMed]

4. Testa, S.; Fornetti, E.; Fuoco, C.; Sanchez-Riera, C.; Rizzo, F.; Ciccotti, M.; Cannata, S.; Sciarra, T.; Gargioli, C. The War after War: Volumetric Muscle Loss Incidence, Implication, Current Therapies and Emerging Reconstructive Strategies, a Comprehensive Review. Biomedicines 2021, 9, 564. [CrossRef] [PubMed]

5. Shen, O.Y.; Chen, Y.F.; Xu, H.T.; Lee, C.W. The Efficacy of Naive versus Modified Mesenchymal Stem Cells in Improving Muscle Function in Duchenne Muscular Dystrophy: A Systematic Review. Biomedicines 2021, 9, 1097. [CrossRef] [PubMed]

6. Sitzia, C.; Meregalli, M.; Belicchi, M.; Farini, A.; Arosio, M.; Bestetti, D.; Villa, C.; Valenti, L.; Brambilla, P.; Torrente, Y. Preliminary Evidences of Safety and Efficacy of Flavonoids- and Omega 3-Based Compound for Muscular Dystrophies Treatment: A Randomized Double-Blind Placebo Controlled Pilot Clinical Trial. Front. Neurol. 2019, 10, 755. [CrossRef] [PubMed]

7. Dubinin, M.V.; Starinets, V.S.; Talanov, E.Y.; Mikheeva, I.B.; Belosludtseva, N.V.; Serov, D.A.; Tenkov, K.S.; Belosludtseva, E.V.; Belosludtsev, K.N. Effect of the Non-Immunosuppressive MPT Pore Inhibitor Alisporivir on the Functioning of Heart Mitochondria in Dystrophin-Deficient mdx Mice. Biomedicines 2021, 9, 1232. [CrossRef] [PubMed] 